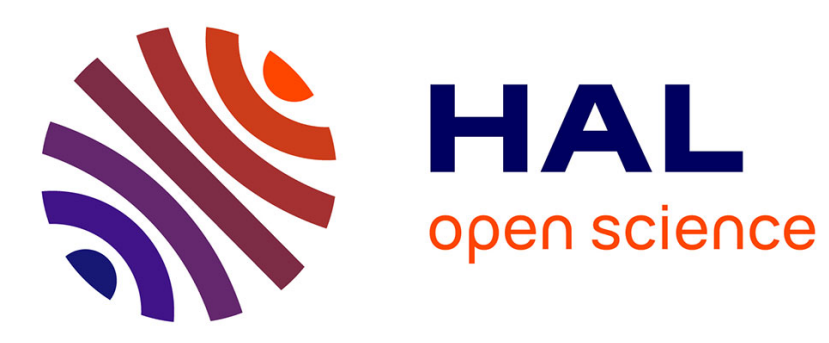

\title{
The Space Frontier: Physical Limits of Multiple Antenna Information Transfer
}

Romain Couillet, Sebastian Wagner, Mérouane Debbah, Alonso Silva

\section{To cite this version:}

Romain Couillet, Sebastian Wagner, Mérouane Debbah, Alonso Silva. The Space Frontier: Physical Limits of Multiple Antenna Information Transfer. International Conference on Performance Evaluation Methodologies and Tools (ValueTools), Oct 2008, Athens, Greece. 10.4108/ICST.VALUETOOLS2008.4574 . hal-01076465

\section{HAL Id: hal-01076465 \\ https://hal.inria.fr/hal-01076465}

Submitted on 22 Oct 2014

HAL is a multi-disciplinary open access archive for the deposit and dissemination of scientific research documents, whether they are published or not. The documents may come from teaching and research institutions in France or abroad, or from public or private research centers.
L'archive ouverte pluridisciplinaire HAL, est destinée au dépôt et à la diffusion de documents scientifiques de niveau recherche, publiés ou non, émanant des établissements d'enseignement et de recherche français ou étrangers, des laboratoires publics ou privés. 


\section{The Space Frontier: Physical Limits of Multiple Antenna Information Transfer}

\author{
Romain Couillet \\ NXP Semiconductors \\ 505, Route des Lucioles \\ Sophia Antipolis \\ 06560 Valbonne, France \\ romain.couillet@supelec.fr
}

\author{
Sebastian Wagner \\ NXP Semiconductors \\ 505, Route des Lucioles \\ Sophia Antipolis \\ 06560 Valbonne, France \\ sebastian.wagner \\ external@nxp.com \\ Alonso Silva \\ Supélec \\ Plateau de Moulon \\ 3, Rue Joliot-Curie \\ 91192 Gif sur Yvette \\ France \\ alonso.silva@supelec.fr
}

\author{
Mérouane Debbah \\ Supélec \\ Plateau de Moulon \\ 3, Rue Joliot-Curie \\ 91192 Gif sur Yvette \\ France
}

merouane.debbah@supelec.fr

\begin{abstract}
In this paper, we study the capacity limits of dense multiantenna systems. We derive asymptotic capacity expressions for point-to-point and broadcast channels by applying recent tools from random matrix theory. In the case of broadcast channels, we focus on linear precoding techniques. We found that the asymptotic capacity depends only on the ratio between the size of the antenna array and the wavelength. This provides useful guidelines on the achievable limits of information transfer. In particular, it is shown that the total capacity grows unbounded if the transmitter has perfect knowledge of the channel, while the capacity saturates in the absence of channel knowledge at the transmitter. We provide numerical results supporting the theoretical derivations.
\end{abstract}

\section{INTRODUCTION}

Sixty years ago, Shannon [1] provided a mathematical framework to analyze fundamental limits of information transfer in the case of single-input single-output channels. He introduced the channel capacity as the maximum rate at which information can be reliably transmitted. From a purely theoretical point of view, there is no bound on the capacity as both bandwidth and power can be arbitrarily high. However, in practice, we can only transmit with finite power and over a restricted frequency band for physical reasons. Recently multiple-input multiple-output (MIMO) systems have been extensively studied since significant growth in terms of

Permission to make digital or hard copies of all or part of this work for personal or classroom use is granted without fee provided that copies are not made or distributed for profit or commercial advantage and that copies bear this notice and the full citation on the first page. To copy otherwise, to republish, to post on servers or to redistribute to lists, requires prior specific permission and/or a fee.

Inter-Perf 2008, October 24, Athens, GREECE.

Copyright , 2008 ICST ISBN \# 978-963-9799-31-8. capacity has been predicted in [2], [3]. More specifically, in a system with $n_{\mathrm{T}}$ transmit and $n_{\mathrm{R}}$ receive antennas the capacity scales linearly with $\min \left(n_{\mathrm{T}}, n_{\mathrm{R}}\right)$ for independent and identically distributed (i.i.d.) Gaussian channels, at high signal-to-noise ratio (SNR). Again, MIMO systems suggest that the capacity can increase to infinity if the number of antennas grows large at both transmitter and receiver.

However, recent works [18] have shown that the capacity, even for an increasing number of antennas, is limited by the density of scatterers in the environment. In other words, the number of antennas should be less than the number of degrees of freedom (modes) provided by the channel. Our goal is to show that, even when the channel offers an infinite number of modes, the capacity is mainly limited by the ratio between the size of the antenna array and the wavelength, which we call the space frontier. Indeed, in general, for a given space, increasing $n_{\mathrm{T}}$ or $n_{\mathrm{R}}$ decreases the relative distances between the antennas. Once the distance is less than half the transmit signal wavelength $\lambda$ the antennas become correlated [16] and the capacity does not grow linearly anymore. In case of a circular antenna array it has been demonstrated by Pollock [4] that the capacity saturates if the number of antennas increases. In this work, we aim to extend Pollock's contribution to one- and twodimensional antenna arrays. We study the capacity limits of point-to-point MIMO channels as well as of MIMO Gaussian broadcast channels (MIMO-GBC) with linear precoding. In the latter we assume a single transmitter modeled as a dense line of antennas which transmits to many independent single-antenna receivers. The general capacity solutions for those schemes are mathematically involved [9] and require the application of recent results from random matrix theory (RMT) and free probability [14].

This paper is organized as follows: Section 2 briefly introduces important tools from random matrix theory. Section 3 presents the capacity limits for the different MIMO channels. Section 4 gives simulation results validating our theoretical claims. Section 5 discusses the theoretical and practical implications of our results. Finally, section 6 states 
our conclusions.

Notation: In the following, boldface lower-case symbols represent vectors, capital boldface characters denote matrices $\left(\mathbf{I}_{N}\right.$ is the $N \times N$ identity matrix). The Hermitian transpose is denoted $(\cdot)^{\mathrm{H}}$. The set of $N \times M$ matrices over the algebra $\mathcal{A}$ is denoted $\mathcal{M}(\mathcal{A}, N, M)$. The operators $\operatorname{det}(\mathbf{X})$ and $\operatorname{tr}(\mathbf{X})$ represent the determinant and the trace of matrix $\mathbf{X}$, respectively. The symbol E[.] denotes expectation. The derivative of a function $f$ of a single variable $x$ is denoted $\frac{\mathrm{d}}{\mathrm{d} x} f$.

\section{RANDOM MATRIX THEORY TOOLS}

Since the pioneering work of Wigner [19] on the asymptotic empirical eigenvalue distribution of random hermitian matrices, random matrix theory has grown into a new field of research in theoretical physics and applied probability. The main application to communications lies in the derivation of asymptotic results for large matrices. Specifically, the eigenvalue distribution of large Hermitian matrices converges, in many practical cases, to a definite probability distribution, called empirical distribution. For instance, if $\mathbf{X} \in \mathcal{M}(\mathbb{C}, N, L)$ is a Gaussian matrix (i.e. a matrix with Gaussian i.i.d. entries), the eigenvalue distribution of the matrix $\frac{1}{L} \mathbf{X X}^{\mathrm{H}}$ is known to converge, when $N, L \rightarrow \infty$ and $N / L \rightarrow c$, towards the Marčhenko-Pastur law $\mu_{c}$ [14].

RMT provides many tools to handle the empirical distribution of large random matrices. Among those tools, the Stieltjes transform $\mathcal{S}_{\mathbf{X}}$ of a large Hermitian matrix $\mathbf{X}$, defined on the half complex space $\{z \in \mathbb{C}, \operatorname{Im}(z)>0\}$, is

$$
\mathcal{S}_{\mathbf{X}}(z)=\int_{-\infty}^{+\infty} \frac{1}{\lambda-z} f(\lambda) \mathrm{d} \lambda
$$

where $f$ is the empirical distribution of $\mathbf{X}$.

Silverstein [13] derived a fixed-point expression of the Stieltjes transform for a particular random matrix structure in the following theorem,

TheOREM 1. Let the entries of the $N \times K$ matrix $\mathbf{W}$ be i.i.d. with zero mean and variance $1 / N$. Let $\mathbf{X}$ be an $N \times N$ Hermitian random matrix with an empirical eigenvalue distribution function converging weakly to $P_{\mathbf{X}}(x)$ almost surely.

Moreover, let $\mathbf{Y}$ be a $K \times K$ real diagonal random matrix with an empirical distribution function converging almost surely in distribution to a probability distribution function $P_{\mathbf{Y}}(x)$ as $K \rightarrow \infty$. Then almost surely, the empirical eigenvalue distribution of the random matrix:

$$
\mathbf{H}=\mathbf{X}+\mathbf{W} \mathbf{Y} \mathbf{W}^{\mathbf{H}}
$$

converges weakly, as $K, N \rightarrow \infty$ but $K / N \rightarrow \alpha$ fixed, to the unique distribution function whose Stieltjes transform satisfies:

$$
\mathcal{S}_{\mathbf{H}}(z)=\mathcal{S}_{\mathbf{X}}\left(z-\alpha \int \frac{y}{1+y \mathcal{S}_{\mathbf{H}}(z)} \mathrm{d} P_{\mathbf{Y}}(y)\right)
$$

This theorem is generalized by Girko [10] who derived a fixed-point equation for the Stieltjes transform of large Hermitian matrices $\mathbf{H}=\mathbf{W} \mathbf{W}^{\mathrm{H}}$ when $\mathbf{W}$ has independent entries $w_{i j}$ with variance $\sigma_{i j}^{2} / N$ such that the set $\left\{\sigma_{i j}^{2}\right\}_{i, j}$ is uniformly upper-bounded. In the following, we will extensively use this result to derive the asymptotic MIMO capacity.

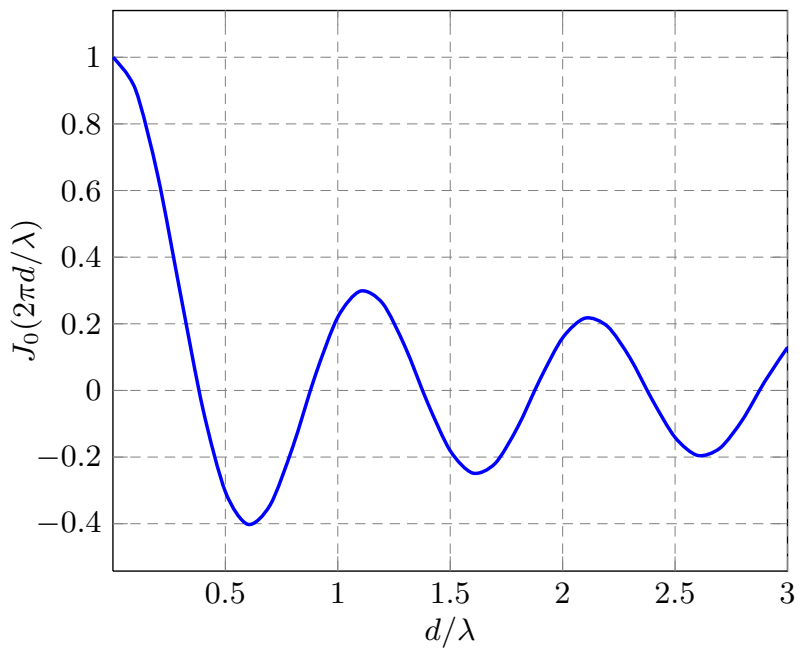

Figure 1: Spatial correlation vs. $d / \lambda$

\section{FUNDAMENTAL CAPACITY LIMITS}

\subsection{Dense MIMO capacity}

We first consider a point-to-point MIMO system with $n_{\mathrm{T}}$ transmit antennas and $n_{\mathrm{R}}$ receive antennas. The linear transmission model is

$$
\mathbf{y}=\sqrt{\frac{\rho}{n_{\mathrm{T}}}} \mathbf{H} \mathbf{x}+\mathbf{n}
$$

with transmit vector $\mathbf{x} \in \mathbb{C}^{n_{\mathrm{T}}}$, receive vector $\mathbf{y} \in \mathbb{C}^{n_{\mathrm{R}}}$ and channel $\mathbf{H} \in \mathcal{M}\left(\mathbb{C}, n_{\mathrm{R}}, n_{\mathrm{T}}\right)$. The noise vector $\mathbf{n}$ has independent circularly symmetric standard Gaussian entries and $\rho$ is the average SNR.

Let the elements of the transmit vector $\mathbf{x}$ be Gaussian with covariance matrix $\mathrm{E}\left[\mathbf{x x}^{\mathrm{H}}\right]=\mathbf{\Phi}$. The ergodic channel capacity is given by [1]

$$
\mathcal{C}\left(n_{\mathrm{R}}, n_{\mathrm{T}}\right)=\mathrm{E}\left[\log \operatorname{det}\left(\mathbf{I}_{n_{\mathrm{R}}}+\frac{\rho}{n_{\mathrm{T}}} \mathbf{H} \mathbf{\Phi} \mathbf{H}^{\mathrm{H}}\right)\right]
$$

Following Jakes' model [12], the spatial autocorrelation functions of fading processes $h_{1}$ and $h_{2}$ experienced by two antennas separated by distance $d$ reads

$$
\mathrm{E}\left[h_{1} h_{2}^{*}\right]=J_{0}(2 \pi d / \lambda)
$$

where $\lambda=c / f_{c}$ denotes the transmit signal wavelength and $J_{0}(x)$ is the zero-order Bessel function of the first kind. Thus, by placing several antennas in close proximity their signals tend to be, to some extent, correlated. The correlation function of Jakes' model is depicted in figure 1.

In [4], Pollock et al. considered an increasing number of antennas distributed on a uniform circular array of fixed radius. By using bounds on the Bessel function, Pollock derived an approximation of the channel capacity and shows that the capacity bound is independent of $\left(n_{\mathrm{R}}, n_{\mathrm{T}}\right)$. In the following, we will extend these results using RMT. For a given $\beta \in \mathbb{R}^{+}$, we will consider that $n_{\mathrm{T}} / n_{\mathrm{R}} \rightarrow \beta$ when $n_{\mathrm{T}}$ and $n_{\mathrm{R}}$ grow large. The entries of $\mathbf{H}$ represent the fading coefficients between each transmit and each receive antenna normalized such that

$$
\mathrm{E}\left[\operatorname{tr}\left(\mathbf{H H}^{\mathrm{H}}\right)\right]=n_{\mathrm{R}} n_{\mathrm{T}}
$$


while

$$
\mathrm{E}\left[\|\mathbf{x}\|^{2}\right]=n_{\mathrm{T}}
$$

It is useful to decompose the input covariance matrix $\mathbf{\Phi}=$ $E\left[\mathbf{x x}^{\mathrm{H}}\right]$ in its eigenvectors and eigenvalues,

$$
\boldsymbol{\Phi}=\mathbf{V P} \mathbf{V}^{\mathrm{H}}
$$

According to the maximum entropy principle [5], the most appropriate density function for $\mathbf{H}$, given $n_{\mathrm{R}}, n_{\mathrm{T}}, l$ and $\lambda$, is the classical separable (also termed Kronecker or product) correlation model [6]

$$
\mathbf{H}=\boldsymbol{\Theta}_{\mathrm{R}}^{1 / 2} \mathbf{H}_{w} \Theta_{\mathrm{T}}^{1 / 2}
$$

where the deterministic matrices $\boldsymbol{\Theta}_{\mathrm{T}}$ and $\boldsymbol{\Theta}_{\mathrm{R}}$ represent the correlation between the transmit antennas and the receive antennas, respectively. The entries of $\mathbf{H}_{w}$ are i.i.d. standard Gaussian. With statistical channel state information at the transmitter (CSIT), capacity is achieved if the eigenvectors of the input covariance $\boldsymbol{\Phi}$ coincide with those of $\boldsymbol{\Theta}_{\mathrm{T}}$. Consequently, denoting $\boldsymbol{\Lambda}_{\mathrm{T}}$ and $\boldsymbol{\Lambda}_{\mathrm{R}}$ the diagonal eigenvalue matrices of $\Theta_{\mathrm{T}}$ and $\Theta_{\mathrm{R}}$ respectively we have

$\mathcal{C}(\beta, \rho)=\lim _{n_{\mathrm{T}} \rightarrow \infty} \log \operatorname{det}\left(\mathbf{I}+\frac{\rho}{n_{\mathrm{T}}} \boldsymbol{\Lambda}_{\mathrm{R}}^{1 / 2} \mathbf{H}_{w} \boldsymbol{\Lambda}_{\mathrm{T}}^{1 / 2} \mathbf{P} \boldsymbol{\Lambda}_{\mathrm{T}}^{1 / 2} \mathbf{H}_{w}^{\mathrm{H}} \boldsymbol{\Lambda}_{\mathrm{R}}^{1 / 2}\right)$

As a direct consequence of theorem 1 :

THEOREM 2. [11] The capacity of a Rayleigh-faded channel with separable transmit and receive correlation matrices $\Theta_{\mathrm{T}}$ and $\Theta_{\mathrm{R}}$ and statistical CSIT almost surely converges to

$$
\begin{aligned}
\frac{\mathcal{C}(\beta, \rho)}{n_{\mathrm{R}}} \rightarrow & \beta \mathrm{E}\left[\log \left(1+\rho \cdot \lambda_{\mathrm{T}} \Gamma(\rho)\right)\right] \\
& +\mathrm{E}\left[\log \left(1+\rho \cdot \lambda_{\mathrm{R}} \Upsilon(\rho)\right)\right] \\
& -\beta \cdot \rho \cdot \Gamma(\rho) \Upsilon(\rho) \log e
\end{aligned}
$$

where

$$
\begin{aligned}
\Gamma(\rho) & =\frac{1}{\beta} \mathrm{E}\left[\frac{\lambda_{\mathrm{R}}}{1+\rho \cdot \lambda_{\mathrm{R}} \Upsilon(\rho)}\right] \\
\Upsilon(\rho) & =\mathrm{E}\left[\frac{\lambda_{\mathrm{T}}}{1+\rho \cdot \lambda_{\mathrm{T}} \Gamma(\rho)}\right]
\end{aligned}
$$

and the dumb random variables $\lambda_{\mathrm{R}}, \lambda_{\mathrm{T}}$ are asymptotically distributed as the diagonal elements of $\boldsymbol{\Lambda}_{\mathrm{R}}$ and $\mathbf{P} \boldsymbol{\Lambda}_{\mathrm{T}}$ respectively.

\subsection{Antenna array geometry and correlation}

\subsubsection{One-dimensional setup}

The antenna setup is depicted in figure 2 . We consider two uniform linear antenna arrays of length $l$ placed at a distance $L$. The transmit and receive array is equipped with $n_{\mathrm{T}}$ and $n_{\mathrm{R}}$ antennas, respectively. The correlation matrices $\boldsymbol{\Theta}_{\mathrm{T}}$ and $\Theta_{\mathrm{R}}$ have the same form and read

$$
\left[\begin{array}{ccccc}
1 & J_{0}\left(\frac{2 \pi}{\lambda} \frac{l}{N-1}\right) & J_{0}\left(\frac{2 \pi}{\lambda} \frac{2 l}{N-1}\right) & \ldots & J_{0}\left(\frac{2 \pi}{\lambda} \frac{(N-1) l}{N-1}\right) \\
J_{0}\left(\frac{2 \pi}{\lambda} \frac{l}{N-1}\right) & 1 & J_{0}\left(\frac{2 \pi}{\lambda} \frac{l}{N-1}\right) & \ldots & J_{0}\left(\frac{2 \pi}{\lambda} \frac{(N-2) l}{N-1}\right) \\
J_{0}\left(\frac{2 \pi}{\lambda} \frac{2 l}{N-1}\right) & J_{0}\left(\frac{2 \pi}{\lambda} \frac{l}{N-1}\right) & 1 & \ldots & \vdots \\
\vdots & \vdots & \vdots & \ddots & \vdots \\
J_{0}\left(\frac{2 \pi}{\lambda} \frac{(N-1) l}{N-1}\right) & J_{0}\left(\frac{2 \pi}{\lambda} \frac{(N-2) l}{N-1}\right) & \ldots & \ldots & 1
\end{array}\right]
$$

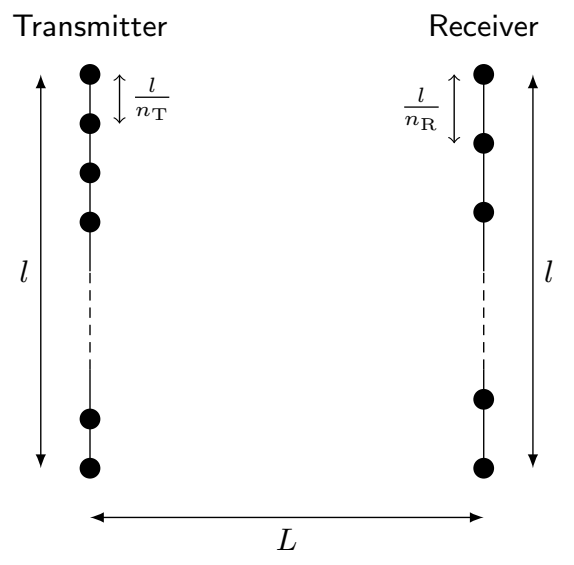

Figure 2: One-dimensional antenna array geometry

with $N$ equal to $n_{\mathrm{T}}, n_{\mathrm{R}}$ for $\boldsymbol{\Theta}_{\mathrm{T}}, \boldsymbol{\Theta}_{\mathrm{R}}$, respectively. The normalized matrices $\frac{1}{n_{\mathrm{R}}} \boldsymbol{\Theta}_{\mathrm{R}}$ and $\frac{1}{n_{\mathrm{T}}} \boldsymbol{\Theta}_{\mathrm{T}}$ are Wiener class Toeplitz matrices [20], i.e.

$$
\lim _{N \rightarrow \infty} \frac{1}{N} \sum_{k=1}^{N}\left|[\boldsymbol{\Theta}]_{1, k}\right|<\infty
$$

There is no exact expression for the eigenvalues like in the case of a circulant matrix. However, for large $\left(n_{\mathrm{R}}, n_{\mathrm{T}}\right)$ the eigenvalue distribution of a Wiener class Toeplitz matrix converges to that of the circulant matrix, both with the same first row [20]. The set of the eigenvalues of $\frac{1}{n_{\mathrm{R}}} \boldsymbol{\Theta}_{\mathrm{R}}$ and $\frac{1}{n_{\mathrm{T}}} \boldsymbol{\Theta}_{\mathrm{T}}$ for large $\left(n_{\mathrm{R}}, n_{\mathrm{T}}\right)$ is the image of the function

$$
\begin{aligned}
F_{1}: \mathbb{N} & \rightarrow \mathbb{R} \\
n & \mapsto \lim _{N \rightarrow \infty} \frac{1}{N} \sum_{p=-(N-1)}^{N-1} J_{0}\left(\frac{2 \pi l}{\lambda} \frac{p}{N-1}\right) \cos \left(2 \pi n \frac{p}{N}\right) \\
& =2 \int_{-1}^{1} J_{0}\left(\frac{2 \pi l}{\lambda} x\right) \cos (2 \pi n x) \mathrm{d} x
\end{aligned}
$$

Since $F(\mathbb{N})$ is a discrete countable set (and not a continuum), the limit eigenvalue distribution of $\boldsymbol{\Theta}_{\mathrm{T}}$ and $\boldsymbol{\Theta}_{\mathrm{R}}$ is a sum of Dirac functions

$$
p_{\nu}(\nu)=\lim _{N \rightarrow \infty} \frac{1}{N} \sum_{k=0}^{N} \delta\left(\nu-N \cdot F_{1}(k)\right)
$$

At this point it is important to note that the cumulated surface of both antenna arrays must be constant regardless of $n_{\mathrm{R}}$ and $n_{\mathrm{T}}$. Hence increasing the number of antennas must lead to a reduction of the individual antenna surface. As a result, the power per receive antenna must scale with $1 / n_{\mathrm{R}}$, hence

$$
\rho=\frac{\rho^{\prime}}{n_{\mathrm{R}}}
$$

for a constant total SNR $\rho^{\prime}$. We first consider the case where no CSIT is available, hence a uniform power allocation over the transmit antennas is optimal (i.e. $\mathbf{P}=\mathbf{I}_{n_{\mathrm{T}}}$ ). Applying theorem 2 and expanding the expectations for large 
$\left(n_{\mathrm{R}}, n_{\mathrm{T}}\right)$, we have

$$
\begin{aligned}
\mathcal{C}\left(\beta, \rho^{\prime}\right)= & \beta n_{\mathrm{R}} \frac{1}{n_{\mathrm{T}}} \sum_{k=0}^{n_{\mathrm{T}}} \log \left(1+\frac{\rho^{\prime}}{n_{\mathrm{R}}} n_{\mathrm{T}} F_{1}(k) \Gamma\right) \\
& +n_{\mathrm{R}} \frac{1}{n_{\mathrm{R}}} \sum_{k=0}^{n_{\mathrm{R}}} \log \left(1+\frac{\rho^{\prime}}{n_{\mathrm{R}}} n_{\mathrm{R}} F_{1}(k) \Upsilon\right) \\
& -n_{\mathrm{R}} \beta \cdot \frac{\rho^{\prime}}{n_{\mathrm{R}}} \cdot \Gamma\left(\rho^{\prime}\right) \Upsilon\left(\rho^{\prime}\right) \log (e)
\end{aligned}
$$

with

$$
\begin{aligned}
& \Gamma\left(\rho^{\prime}\right)=\frac{1}{\beta n_{\mathrm{R}}} \sum_{k=0}^{n_{\mathrm{R}}} \frac{n_{\mathrm{R}} F_{1}(k)}{1+\rho^{\prime} F_{1}(k) \Upsilon} \\
& \Upsilon\left(\rho^{\prime}\right)=\frac{1}{n_{\mathrm{T}}} \sum_{k=0}^{n_{\mathrm{T}}} \frac{n_{\mathrm{T}} F_{1}(k)}{1+\rho^{\prime} \beta F_{1}(k) \Gamma}
\end{aligned}
$$

In the limit, this becomes

$$
\begin{aligned}
\mathcal{C}\left(\beta, \rho^{\prime}\right) \rightarrow & \sum_{k=0}^{+\infty} \log \left(1+\rho^{\prime} \beta F_{1}(k) \Gamma\right) \\
& +\sum_{k=0}^{+\infty} \log \left(1+\rho^{\prime} F_{1}(k) \Upsilon\right) \\
& -\beta \rho^{\prime} \Gamma\left(\rho^{\prime}\right) \Upsilon\left(\rho^{\prime}\right) \log (e)
\end{aligned}
$$

with

$$
\begin{aligned}
& \Gamma\left(\rho^{\prime}\right)=\frac{1}{\beta} \sum_{k=0}^{+\infty} \frac{F_{1}(k)}{1+\rho^{\prime} F_{1}(k) \Upsilon} \\
& \Upsilon\left(\rho^{\prime}\right)=\sum_{k=0}^{+\infty} \frac{F_{1}(k)}{1+\rho^{\prime} \beta F_{1}(k) \Gamma}
\end{aligned}
$$

where $\forall k \in \mathbb{N}, F_{1}(k) \geq 0$ as they are eigenvalues of a covariance matrix. Also $\sum_{k=0}^{+\infty} F_{1}(k)=\frac{1}{n_{\mathrm{R}}} \operatorname{tr}\left(\Theta_{\mathrm{R}}\right)=1$. This implies that $\Gamma$ and $\Upsilon$ are finite and therefore the total capacity $\mathcal{C}$ is also finite.

Further note that (19) only depends on the system parameters through the ratio $l / \lambda$. This leads to the conclusion that the MIMO capacity limit depends only on the ratio $l / \lambda$ and $\beta$ when CSIT is absent.

Consider now the case of perfect CSIT. Here, it is optimal to distribute the power according to the water-filling solution [21]. That is, only sufficiently strong eigenmodes of the channel (10) are used for transmission. If we allocate the power constrained by (8) on the dominating channel eigenmodes (i.e. the relevant eigenvalues of $\frac{1}{n_{\mathrm{T}}} \boldsymbol{\Theta}_{\mathrm{R}}^{1 / 2} \mathbf{H}_{w} \boldsymbol{\Theta}_{\mathrm{T}} \mathbf{H}_{w}^{\mathrm{H}} \boldsymbol{\Theta}_{\mathrm{R}}^{1 / 2}$ ), then for large $n_{\mathrm{T}}$, the capacity grows unbounded. As a result, increasing the number of antennas at either side of the transmission allows to achieve arbitrarily high capacity under the assumption of perfect CSIT.

\subsubsection{Two-dimensional setup}

The previous scheme can be extended to a surface area. We now increase the density of antennas uniformly along each dimension of the surface. Consider a rectangular surface of respective height and width $l_{x}$ and $l_{y}$. Then, equation (19) has an equivalent version in two dimensions,

$$
p_{\nu}(\nu)=\lim _{N \rightarrow \infty} \frac{1}{N} \sum_{k=0}^{N} \delta\left(\nu-N \cdot F_{2}(k)\right)
$$

with

$$
\begin{aligned}
F_{2}(k)= & 2 \int_{-l_{x}}^{l_{x}} \int_{-l_{y}}^{l_{y}} J_{0}\left(\frac{2 \pi l_{x}}{\lambda} \sqrt{u_{x}^{2}+\left(\frac{l_{y}}{l_{x}}\right)^{2} u_{y}^{2}}\right) \\
& \times \cos \left(2 \pi \nu\left(u_{x}+u_{y}\right)\right) \mathrm{d} u_{x} \mathrm{~d} u_{y}
\end{aligned}
$$

From (27), one verifies that the final capacity formulation depends on the two constant values $l_{x} / \lambda$ and $l_{x} / l_{y}$, or similarly on the two ratios $l_{x} / \lambda$ and $l_{y} / \lambda$. Note that the capacity for a given surface might then differ depending on the shape of the surface.

\subsection{MIMO-GBC capacity}

We assume a GBC generated by a multi-antenna transmitter and many non-cooperative single-antenna receivers. It has been shown in [8] that the capacity region is achieved by dirty-paper coding (DPC). However, to derive closedform expressions, we restrict our analysis in the following to suboptimal linear precoding techniques. As the receivers are uncorrelated, the GBC channel model is

$$
\mathbf{H}=\mathbf{H}_{w} \Theta_{\mathrm{T}}^{1 / 2}
$$

and the transmitted signal $\mathbf{x}$ is obtained by

$$
\mathbf{x}=\mathbf{G u}
$$

where the symbol vector $\mathbf{u} \in \mathbb{C}^{n_{\mathrm{R}}}$ has unit power, and $\mathbf{G} \in$ $\mathcal{M}\left(\mathbb{C}, n_{\mathrm{T}}, n_{\mathrm{R}}\right)$.

\subsubsection{ZF-beamforming}

Zero-Forcing (ZF) beamforming is a mere channel inversion precoding. With the same notation as in previous sections, the channel model reads

$$
\mathbf{y}=\sqrt{\frac{\rho}{n_{\mathrm{T}}}} \mathbf{H} \mathbf{x}+\mathbf{n}
$$

with

$$
\mathbf{x}= \begin{cases}\alpha \mathbf{H}^{\prime-1} \mathbf{u} & , \text { if } \beta=1 \\ \alpha\left(\mathbf{H}^{\prime{ }^{\mathrm{H}}} \mathbf{H}^{\prime}\right)^{-1} \mathbf{H}^{\prime \mathrm{H}} \mathbf{u} & , \text { if } \beta>1\end{cases}
$$

for $\mathbf{H}^{\prime}=\sqrt{\frac{1}{n_{\mathrm{T}}}} \mathbf{H}$. The parameter $\alpha$ is set to fulfill the transmission power constraint (8) which leads to

$$
\alpha^{2}=\frac{1}{\frac{1}{n_{\mathrm{T}}} \operatorname{tr}\left(\mathbf{H}^{\prime} \mathbf{H}^{\prime \mathbf{H}}\right)^{-1}}
$$

where

$$
\frac{1}{n_{\mathrm{T}}} \operatorname{tr}\left(\mathbf{H}^{\prime} \mathbf{H}^{\prime \mathrm{H}}\right)^{-1} \rightarrow \int \frac{1}{\nu} f(\nu) \mathrm{d} \nu
$$

with $f$ the empirical distribution of $\mathbf{H}^{\prime} \mathbf{H}^{\prime \mathbf{H}}$. We recognize in (34) the Stieltjes transform of $f(x)$ in $x=0$.

In contrast to [22] where no power limitation is imposed on $\mathbf{x}$, no asymptotic expression for $\alpha$ is known when $\left(n_{\mathrm{R}}, n_{\mathrm{T}}\right)$ grow large to the authors' knowledge.

Recall that $\mathbf{H H}^{\mathrm{H}}=\mathbf{H}_{w} \boldsymbol{\Theta}_{\mathrm{T}} \mathbf{H}_{w}^{\mathrm{H}}$. Thus by diagonalizing $\Theta_{\mathrm{T}}=\mathbf{V} \boldsymbol{\Lambda}_{\mathrm{T}} \mathbf{V}^{\mathrm{H}}$ with unitary matrix $\mathbf{V}$, we have

$$
\mathbf{H}^{\prime} \mathbf{H}^{\prime \mathrm{H}}=\left(\frac{1}{\sqrt{n_{\mathrm{T}}}} \mathbf{H}_{w} \mathbf{V}\right) \boldsymbol{\Lambda}_{\mathrm{T}}\left(\frac{1}{\sqrt{n_{\mathrm{T}}}} \mathbf{V}^{\mathrm{H}} \mathbf{H}_{w}^{\mathrm{H}}\right)
$$

where the entries of $\frac{1}{\sqrt{n_{\mathrm{T}}}} \mathbf{H}_{w} \mathbf{V}$ are i.i.d. with zero mean and variance $\frac{1}{n_{\mathrm{T}}}$, and $\boldsymbol{\Lambda}_{\mathrm{T}}$ is distributed as in (19). Applying 
theorem 1 , we prove the existence of $\mathcal{S}_{\mathbf{H}^{\prime} \mathbf{H}^{\prime}}$, when $n_{\mathrm{T}} / n_{\mathrm{R}} \rightarrow$ $\beta$, that satisfies

$$
\mathcal{S}_{\mathbf{H}^{\prime} \mathbf{H}^{\prime \mathbf{H}}}(z)=\left(-z+\beta \int \frac{\nu p_{\nu}(\nu)}{1+\nu \cdot \mathcal{S}_{\mathbf{H}^{\prime} \mathbf{H}^{\prime \mathbf{H}}}(z)} \mathrm{d} \nu\right)^{-1}
$$

Expanding $\mathbf{x}$ according to (4), one obtains parallel noninterfering channels with the per-user capacity

$$
\begin{aligned}
\mathcal{C}_{u}(\beta, \rho) & =\log \left(1+\rho \alpha^{2}\right) \\
& =\log \left(1+\rho \mathcal{S}_{\mathbf{H}^{\prime} \mathbf{H}^{\prime \mathbf{H}}}(0)^{-1}\right)
\end{aligned}
$$

In our specific correlation scenario, this capacity limit is in fact zero. Indeed, if $n_{\mathrm{T}}=n_{\mathrm{R}}$ we have

$$
\frac{1}{n_{\mathrm{T}}} \operatorname{tr}\left(\mathbf{H}^{\prime} \mathbf{H}^{\prime \mathrm{H}}\right)^{-1}=\frac{1}{n_{\mathrm{T}}} \operatorname{tr}\left(\left(\tilde{\mathbf{H}}_{w}^{\mathrm{H}} \tilde{\mathbf{H}}_{w}\right)^{-1} \boldsymbol{\Lambda}_{\mathrm{T}}^{-1}\right)
$$

where $\tilde{\mathbf{H}}_{w}=\mathbf{H}_{w} \mathbf{V}$ is a Gaussian random matrix with entries of variance $1 / n_{\mathrm{T}}$.

Lemma 3. For any two Hermitian $n \times n$ matrices $\mathbf{A}$ and $\mathbf{B}$ with eigenvalues $\lambda_{i}(\mathbf{A})$ and $\lambda_{i}(\mathbf{B})$ respectively arranged in decreasing order,

$$
\operatorname{tr}(\mathbf{A B}) \geq \sum_{i=1}^{n} \lambda_{i}(\mathbf{A}) \lambda_{n-i+1}(\mathbf{B})
$$

From lemma 3, we have

$$
\operatorname{tr}\left(\left(\tilde{\mathbf{H}}_{w}^{\mathrm{H}} \tilde{\mathbf{H}}_{w}\right)^{-1} \boldsymbol{\Lambda}_{\mathrm{T}}^{-1}\right) \geq \sum_{i=1}^{n_{\mathrm{R}}} \lambda_{i}\left(\left(\tilde{\mathbf{H}}_{w}^{\mathrm{H}} \tilde{\mathbf{H}}_{w}\right)^{-1}\right) \lambda_{n-i+1}\left(\boldsymbol{\Lambda}_{\mathrm{T}}^{-1}\right)
$$

The eigenvalues of $\tilde{\mathbf{H}}_{w}^{\mathrm{H}} \tilde{\mathbf{H}}_{w}$ are known [14] to be asymptotically distributed as the Marčhenko-Pastur law on a bounded (positive) support excluding zero. Therefore the eigenvalues of $\left(\tilde{\mathbf{H}}_{w}^{\mathrm{H}} \tilde{\mathbf{H}}_{w}\right)^{-1}$ are also bounded on a finite positive support. Denote $\lambda_{\min }$ the minimum of those eigenvalues, we have

$$
\operatorname{tr}\left(\left(\tilde{\mathbf{H}}_{w}^{\mathrm{H}} \tilde{\mathbf{H}}_{w}\right)^{-1} \boldsymbol{\Lambda}_{\mathrm{T}}^{-1}\right) \geq \lambda_{\min } \sum_{i=1}^{n_{\mathrm{R}}} \lambda_{i}\left(\boldsymbol{\Lambda}_{\mathrm{T}}^{-1}\right)
$$

Observing that

$$
\lambda_{n_{\mathrm{R}}}\left(\boldsymbol{\Lambda}_{\mathrm{T}}\right)=\sum_{p=-\left(n_{\mathrm{R}}-1\right)}^{n_{\mathrm{R}}-1} J_{0}\left(\frac{2 \pi l}{\lambda} \frac{p}{n_{\mathrm{R}}-1}\right) \cos \left(2 \pi \frac{p}{N}\right) \rightarrow 0
$$

we conclude

$$
\operatorname{tr}\left(\left(\tilde{\mathbf{H}}_{w}^{\mathrm{H}} \tilde{\mathbf{H}}_{w}\right)^{-1} \boldsymbol{\Lambda}_{\mathrm{T}}^{-1}\right) \rightarrow+\infty
$$

Therefore, $\alpha^{2} \rightarrow 0$ and the ZF capacity goes to zero for increasing $\left(n_{\mathrm{R}}, n_{\mathrm{T}}\right)$ and $\beta=1$. The case $n_{\mathrm{T}}>n_{\mathrm{R}}$ can be solved by dividing $\tilde{\mathbf{H}}_{w}$ in a two blocks of size $n_{\mathrm{R}} \times n_{\mathrm{R}}$ and $\left(n_{\mathrm{T}}-n_{\mathrm{R}}\right) \times n_{\mathrm{R}}$ where the capacity limit for the former already grows to infinity.

\subsubsection{MMSE-beamforming}

Let us consider regularized ZF-beamforming. The system model in (31) becomes

$$
\mathbf{x}=\left(\mathbf{H}^{\prime{ }^{H}} \mathbf{H}^{\prime}+\alpha \mathbf{I}_{n_{\mathrm{T}}}\right)^{-1} \mathbf{H}^{\prime \mathbf{H}} \mathbf{u}
$$

When $\alpha=0$, we fall back the ZF solution. The parameter $\alpha$ is set so to fulfill the transmission power constraint (8) which leads to

$$
\begin{aligned}
1 & =\frac{1}{n_{\mathrm{T}}} \operatorname{tr}\left(\left(\mathbf{H}^{\prime}{ }^{\mathrm{H}} \mathbf{H}^{\prime}+\alpha \mathbf{I}\right)^{-1} \mathbf{H}^{\prime \mathrm{H}} \mathbf{H}^{\prime}\left(\mathbf{H}^{\prime}{ }^{\mathrm{H}} \mathbf{H}^{\prime}+\alpha \mathbf{I}\right)^{-1}\right) \\
& =\frac{1}{n_{\mathrm{T}}} \operatorname{tr}\left(\left(\mathbf{H}^{\prime}{ }^{\mathrm{H}} \mathbf{H}^{\prime}+\alpha \mathbf{I}\right)^{-2} \mathbf{H}^{\left.\prime{ }^{\mathrm{H}} \mathbf{H}^{\prime}\right)}\right. \\
& \rightarrow \int \frac{\nu}{(\nu+\alpha)^{2}} f(\nu) \mathrm{d} \nu \\
& =\int\left(\frac{1}{(\nu+\alpha)}-\frac{\alpha}{(\nu+\alpha)^{2}}\right) f(\nu) \mathrm{d} \nu \\
& =\mathcal{S}_{\mathbf{H}^{\prime} \mathbf{H}^{\prime \mathbf{H}}}(-\alpha)+\alpha \frac{\mathrm{d}}{\mathrm{d} x} \mathcal{S}_{\mathbf{H}^{\prime} \mathbf{H}^{\prime \mathbf{H}}}(-\alpha)
\end{aligned}
$$

The received signal can be written as

$$
\mathbf{y}=\sqrt{\rho} \cdot \mathbf{H}^{\prime}\left(\mathbf{H}^{\prime \mathrm{H}} \mathbf{H}+\alpha \mathbf{I}\right)^{-1} \mathbf{H}^{\prime \mathrm{H}} \mathbf{u}+\mathbf{n}
$$

Let us denote $\mathbf{H}^{\prime \mathbf{H}}=\left[\mathbf{h}_{1}, \ldots, \mathbf{h}_{n_{\mathrm{R}}}\right]$. We will focus on user $i$ without loss of generality. The received symbol of user $i$ is

$$
\begin{aligned}
y_{i} & =\sqrt{\rho} \cdot \mathbf{h}_{i}^{\mathrm{H}}\left(\mathbf{H}^{\prime \mathrm{H}} \mathbf{H}^{\prime}+\alpha \mathbf{I}\right)^{-1} \mathbf{h}_{i} u_{i} \\
& +\sum_{k=1, k \neq i}^{n_{\mathrm{R}}} \mathbf{h}_{i}^{\mathrm{H}}\left(\mathbf{H}^{\prime \mathrm{H}} \mathbf{H}^{\prime}+\alpha \mathbf{I}\right)^{-1} \mathbf{h}_{k} u_{k} \\
& +\mathbf{n}
\end{aligned}
$$

Lemma 4. [15] Let $\mathbf{A}$ be a deterministic $N \times N$ complex matrix with uniformly bounded spectral radius for all $N$. Let $\mathbf{x}=\frac{1}{\sqrt{N}}\left[x_{1}, \ldots, x_{N}\right]^{T}$ where the $\left\{x_{i}\right\}$ are i.i.d complex random variables with zero mean, unit variance and finite eighth moment. Then

$$
\mathbb{E}\left[\left|\mathbf{x}^{\mathrm{H}} \mathbf{A} \mathbf{x}-\frac{1}{N} \operatorname{tr} \mathbf{A}\right|^{4}\right] \leq \frac{c}{N^{2}}
$$

where $c$ is a constant that does not depend on $N$ or $\mathbf{A}$.

COROLlary 5. This result ensures that

$$
\mathbf{x}^{\mathrm{H}} \mathbf{A} \mathbf{x}-\frac{1}{N} \operatorname{tr} \mathbf{A} \rightarrow 0
$$

almost surely.

Henceforth we write $\mathbf{U}_{i}^{\mathrm{H}}=\left[\mathbf{h}_{1}, \ldots, \mathbf{h}_{i-1}, \mathbf{h}_{i+1}, \ldots, \mathbf{h}_{n_{\mathrm{R}}}\right]$ (in other words, we remove column $i$ ). Applying the matrix inversion lemma yields

$$
\mathbf{h}_{i}^{\mathrm{H}}\left(\mathbf{H}^{\prime \mathrm{H}} \mathbf{H}^{\prime}+\alpha \mathbf{I}\right)^{-1}=\frac{\mathbf{h}_{i}^{\mathrm{H}}\left(\mathbf{U}_{i}^{\mathrm{H}} \mathbf{U}_{i}+\alpha \mathbf{I}\right)^{-1}}{1+\mathbf{h}_{i}^{\mathrm{H}}\left(\mathbf{U}_{i}^{\mathrm{H}} \mathbf{U}_{i}+\alpha \mathbf{I}\right)^{-1} \mathbf{h}_{i}}
$$

As the elements of $\mathbf{h}_{i}$ are i.i.d. (due to the one sided correlation assumption), we can use lemma 4

$$
\mathbf{h}_{i}^{\mathrm{H}}\left(\mathbf{H}^{\prime \mathrm{H}} \mathbf{H}^{\prime}+\alpha \mathbf{I}\right)^{-1} \mathbf{h}_{i} \rightarrow \frac{1}{n_{\mathrm{T}}} \operatorname{tr}\left(\mathbf{H}^{\prime \mathrm{H}^{\mathrm{H}}} \mathbf{H}^{\prime}\right)
$$

Asymptotically, the removal of a single column in the large matrix $\mathbf{H}^{\prime}$ does not affect $\operatorname{tr}\left(\mathbf{H}^{\prime{ }^{H}} \mathbf{H}^{\prime}\right)$, we have

$$
\mathbf{h}_{i}^{\mathrm{H}}\left(\mathbf{U}_{i}^{\mathrm{H}} \mathbf{U}_{i}+\alpha \mathbf{I}\right)^{-1} \mathbf{h}_{i} \rightarrow \mathbf{h}_{i}^{\mathrm{H}}\left(\mathbf{H}^{\prime \mathrm{H}^{\mathrm{H}}} \mathbf{H}^{\prime}+\alpha \mathbf{I}\right)^{-1} \mathbf{h}_{i}
$$

hence

$$
\mathbf{h}_{i}^{\mathrm{H}}\left(\mathbf{H}^{\prime{ }^{\prime H}} \mathbf{H}^{\prime}+\alpha \mathbf{I}\right)^{-1} \rightarrow \frac{\mathbf{h}_{i}^{\mathrm{H}}\left(\mathbf{U}_{i}^{\mathrm{H}} \mathbf{U}_{i}+\alpha \mathbf{I}\right)^{-1}}{1+\mathcal{S}_{\mathbf{H}^{\prime} \mathbf{H}^{\prime \mathbf{H}}}(-\alpha)}
$$




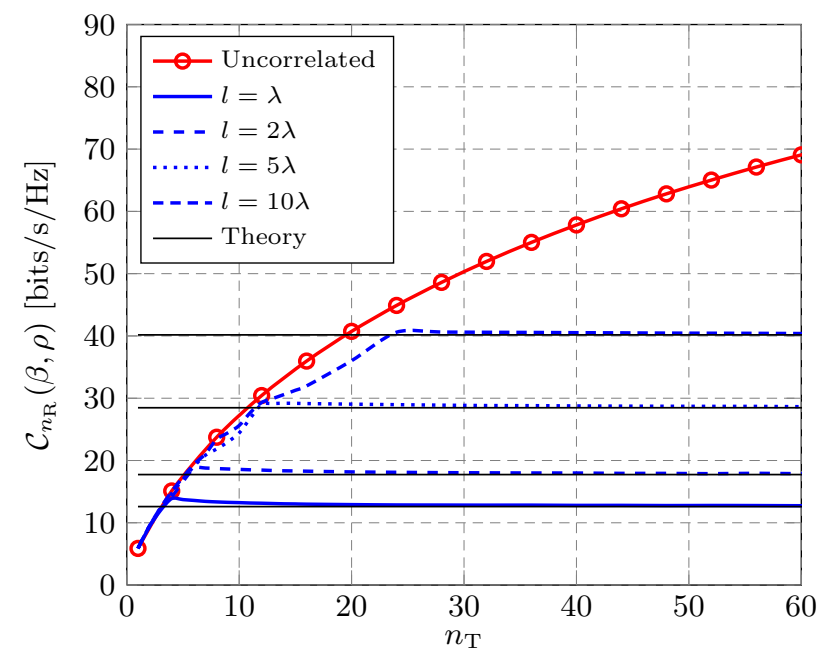

Figure 3: Ergodic point-to-point MIMO capacity $\mathcal{C}_{n_{\mathrm{R}}}(\beta, \rho)$ without CSIT for different $l / \lambda, n_{\mathrm{R}}=n_{\mathrm{T}}$, $\rho^{\prime}=20 \mathrm{~dB}$

Denote $\gamma=\left(1+\mathcal{S}_{\mathbf{H H}^{\mathbf{H}}}(-\alpha)\right)^{-2}$. The expression for the SINR is therefore given by

$$
\operatorname{SINR}_{i}=\frac{\rho \gamma \mathbf{h}_{i}^{\mathrm{H}} \mathbf{W}_{i}^{2} \mathbf{h}_{i}}{\rho \gamma \mathbf{h}_{i}^{\mathrm{H}} \mathbf{W}_{i} \mathbf{U}_{i}^{\mathrm{H}} \mathbf{U}_{i} \mathbf{W}_{i} \mathbf{h}_{i}+1}
$$

with $\mathbf{W}_{i}=\left(\mathbf{U}_{i}^{\mathrm{H}} \mathbf{U}_{i}+\alpha \mathbf{I}\right)^{-1}$. In the limit this leads to a user-independent SINR

$$
\mathrm{SINR} \rightarrow \frac{\rho \gamma \mathcal{S}_{\mathbf{H}^{\prime} \mathbf{H}^{\prime \mathbf{H}}}^{2}(-\alpha)}{\rho \gamma\left(\mathcal{S}_{\mathbf{H}^{\prime} \mathbf{H}^{\prime \mathbf{H}}}(-\alpha)+\alpha \frac{\mathrm{d}}{\mathrm{d} x} \mathcal{S}_{\mathbf{H}^{\prime} \mathbf{H}^{\prime \mathbf{H}}}(-\alpha)\right)+1}
$$

The corresponding per-user capacity is

$$
\mathcal{C}_{u}(\beta, \rho)=\log (1+\operatorname{SINR})
$$

Diagonalizing $\mathbf{U}^{\mathrm{H}} \mathbf{U}$, we observe that the numerator in (57) converges to finite strictly positive values (for the regularization term $\alpha$ ensures that no term diverges). However, as already noted, the strongest eigenvalue of $\boldsymbol{\Theta}_{\mathrm{T}}$ grows linearly with $n_{\mathrm{T}}$, hence, with to lemma 3 , the denominator grows to infinite for large $n_{\mathrm{T}}$. This proves that the per-user capacity goes to zero.

Hence, for large $\left(n_{\mathrm{R}}, n_{\mathrm{T}}\right)$ the MMSE-beamforming algorithm yields zero per-user capacity. Therefore both MMSE beamforming and ZF beamforming achieve asymptotically zero per-user capacity.

As a consequence, it turns out that additional antennas might impair the achievable transmission rate. This is explained by the fact that loading power on more and more correlated antennas, instead of available channel modes, is an inefficient power allocation strategy.

\section{SIMULATION AND RESULTS}

Let us first consider the point-to-point MIMO scenario with dense antenna arrays at both transmitter and receiver side. Figures 3 and 4 present the results of ergodic capacities found by numerical simulation.

$$
\mathcal{C}_{n_{\mathrm{R}}}(\beta, \rho)=n_{\mathrm{R}} \mathrm{E}\left[\log \operatorname{det}\left(\mathbf{I}+\frac{\rho}{n_{\mathrm{T}}} \boldsymbol{\Lambda}_{\mathrm{R}}^{1 / 2} \mathbf{H}_{w} \boldsymbol{\Lambda}_{\mathrm{T}}^{1 / 2} \mathbf{P} \boldsymbol{\Lambda}_{\mathrm{T}}^{1 / 2} \mathbf{H}_{w}^{\mathrm{H}} \boldsymbol{\Lambda}_{\mathrm{R}}^{1 / 2}\right)\right]
$$

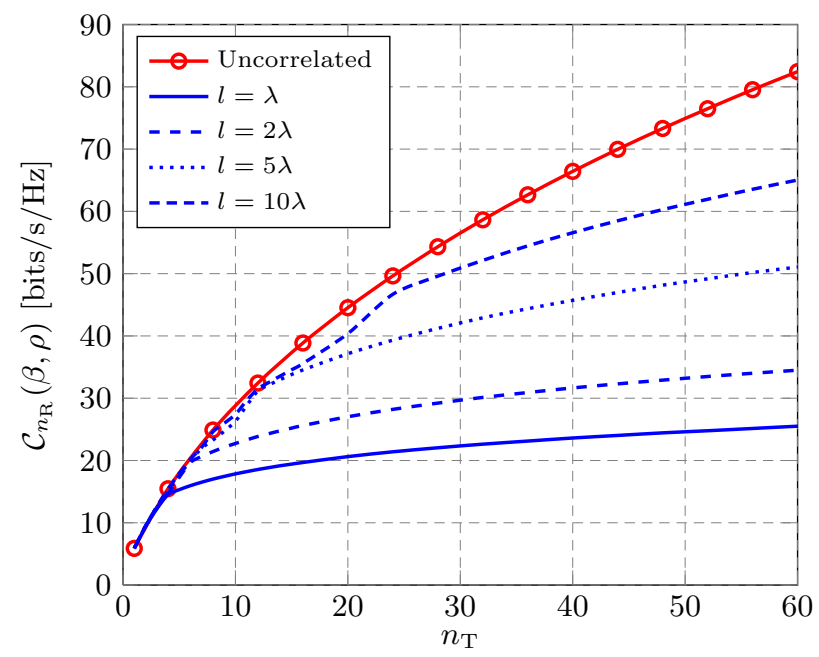

Figure 4: Ergodic point-to-point MIMO capacity $\mathcal{C}_{n_{\mathrm{R}}}(\beta, \rho)$ with perfect CSIT for different $l / \lambda, n_{\mathrm{R}}=n_{\mathrm{T}}$, $\rho^{\prime}=20 \mathrm{~dB}$

In figure 3 , we allocate equal power (i.e. $\mathbf{P}=\mathbf{I}_{n_{\mathrm{T}}}$ ) to the transmit symbols. We observe, as previously concluded, that the capacity saturates for large $\left(n_{\mathrm{T}}, n_{\mathrm{R}}\right)$. In addition we provide the theoretical limits derived from (11) (which are obtained by solving numerically $(25),(26))$. Note that the capacity increases first to a maximum for small $\left(n_{\mathrm{R}}, n_{\mathrm{T}}\right)$ and then decreases to the capacity limit. In figure 4 , we apply water-filling (i.e. loading the transmit power on the dominant eigenmodes of the channel), which leads to a nonsaturating capacity.

Let us now consider the MIMO-GBC with uncorrelated transmitters/receivers and ZF-beamforming. As has been shown in [22] the sum capacity is saturating if $\beta=1$ and growing linearly with $n_{\mathrm{R}}$ when $\beta>1$ which is in accordance with figure 6 . From this figure we further observe that the sum capacity is going to zero in case of correlation between the transmit antennas. Figure 5 shows the corresponding per-user capacity.

In figures 7 and 8 we apply MMSE-beamforming. Since no closed-form solution for $\alpha$ under the constraint (50) is available, the optimal $\alpha$ is found by exhaustive search. We observe again that the per-user capacity is going asymptotically to zero, which is in accordance with equations (58) and (59). The same observation can be made for the sum capacity in figure 8 . Both, the per-user capacity and the sum capacity are decreasing less rapidly for large $\left(n_{\mathrm{R}}, n_{\mathrm{T}}\right)$ than in the case of ZF-beamforming.

\section{DISCUSSION}

A few limitations are worth mentioning about our previous conclusions. In the MIMO case we stated that, with perfect CSIT, the channel capacity grows unbounded even with a strong antenna correlation at the transmitter side. This might indicate that densifying the array of transmit antennas is the preferred option to increase the capacity (rather than increasing the transmitted power or the channel bandwidth). However, perfect CSIT implies that the receiver has to feed back channel information to the transmitter (either as pilot sequences or as directly quantized CSI). For a dense 


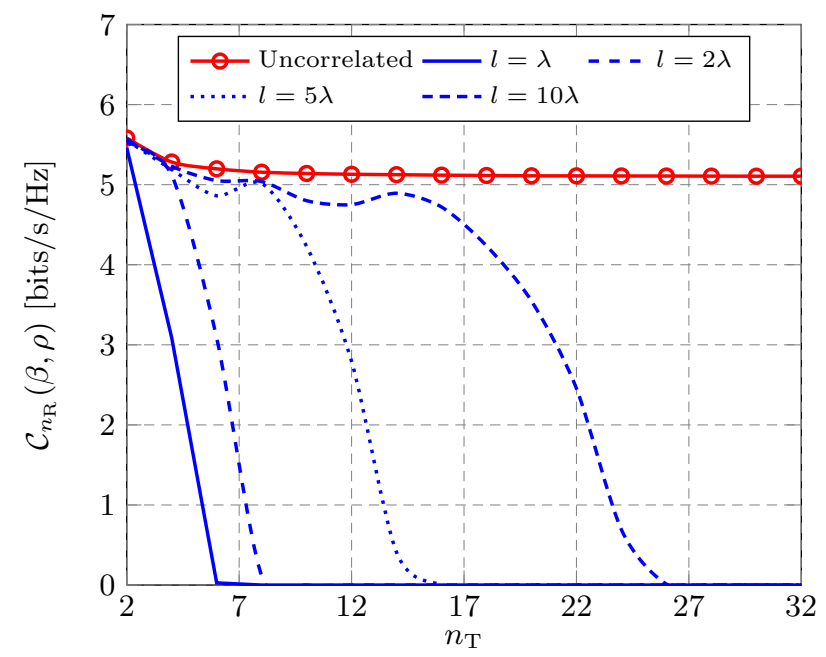

Figure 5: Ergodic MIMO-GBC-ZF per-user capacity $\mathcal{C}_{n_{\mathrm{R}}}(\beta, \rho)$ for different $l / \lambda, n_{\mathrm{T}}=\frac{3}{2} n_{\mathrm{R}}$

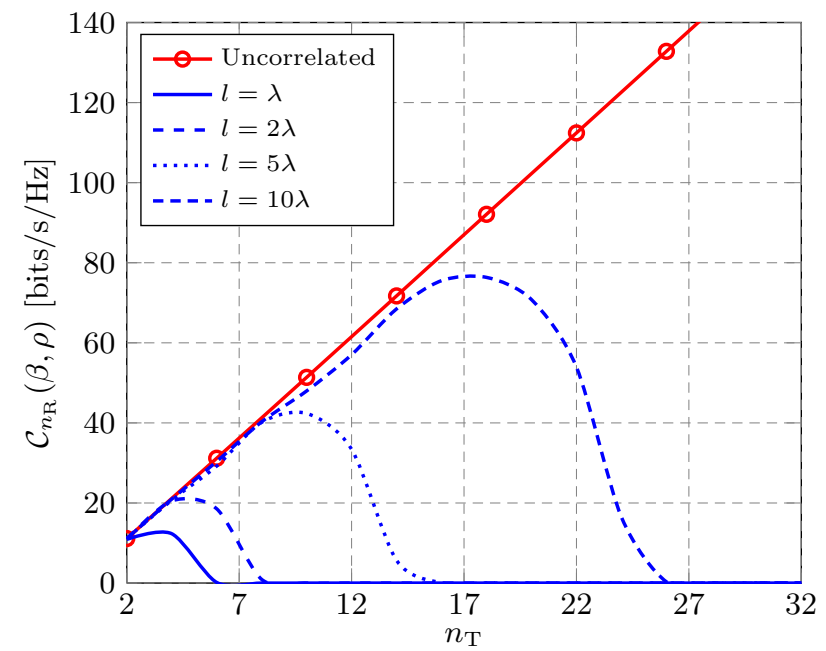

Figure 6: Ergodic MIMO-GBC-ZF sum capacity $n_{\mathrm{R}} \mathcal{C}_{n_{\mathrm{R}}}(\beta, \rho)$ for different $l / \lambda, n_{\mathrm{T}}=\frac{3}{2} n_{\mathrm{R}}$

MIMO system, this introduces an enormous feedback overhead and is thus reducing the achievable throughput.

The same conclusion holds for channel state information at the receiver (CSIR). As Tse demonstrated [17], the capacity with perfect CSIR is limited by the coherence time of the channel. If the number of antennas grows, one needs to estimate more and more degrees of freedom with less power. An optimal trade-off must then be found between increasing the number of antennas (and thus the capacity) and decreasing the amount of channel state information required for reliable transmission. However, if the channel coherence time is infinite and a long synchronization stage prior to data transmission is allowed, then the channel capacity can effectively go unbounded. The only limitation that would appear lies in the physical ability to design a dense array of antennas on a limited surface. In addition, a dense scattering environment is necessary to assure that the assumed channel model is accurate.

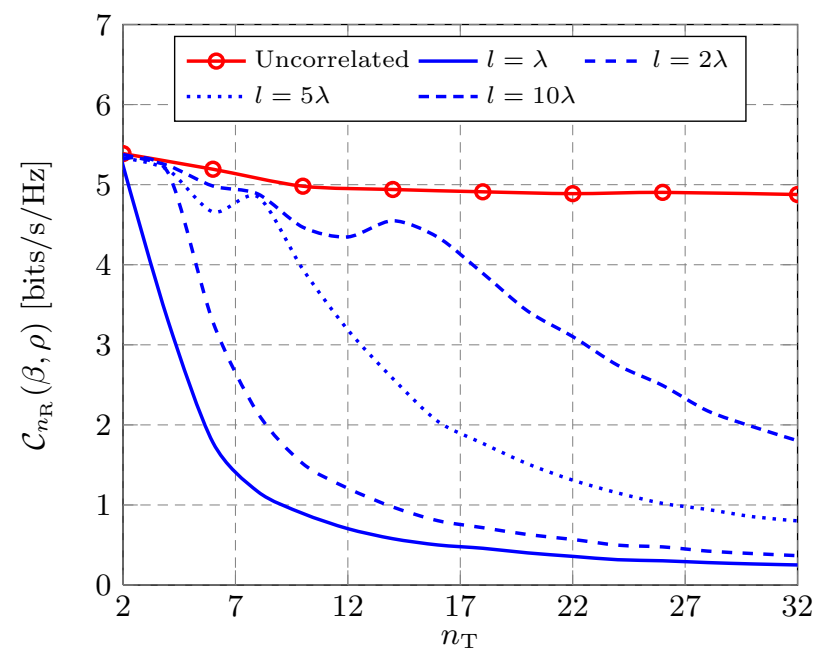

Figure 7: Ergodic MIMO-GBC-MMSE per-user capacity $\mathcal{C}_{n_{\mathrm{R}}}(\beta, \rho)$ for different $l / \lambda, n_{\mathrm{T}}=\frac{3}{2} n_{\mathrm{R}}$

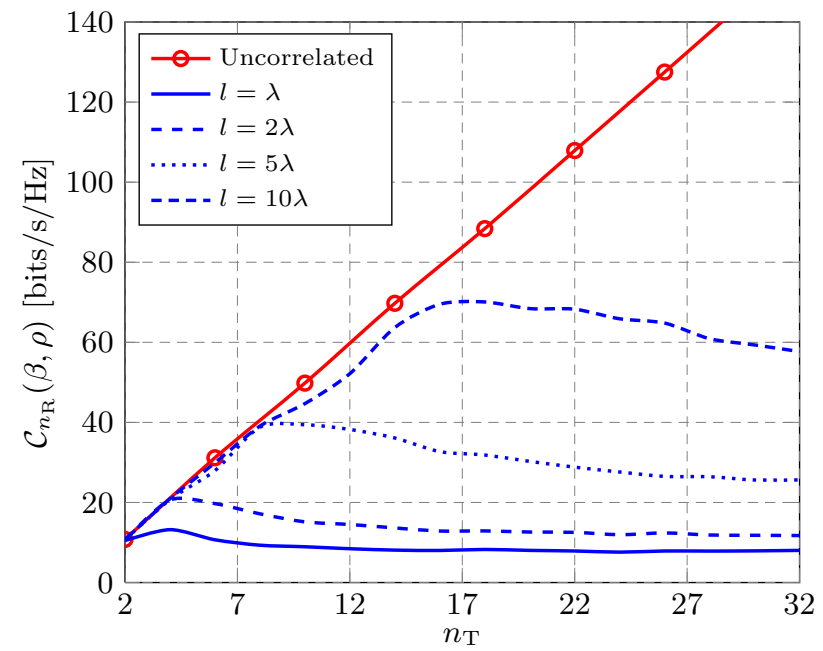

Figure 8: Ergodic MIMO-GBC-MMSE sum capacity $n_{\mathrm{R}} \mathcal{C}_{n_{\mathrm{R}}}(\beta, \rho)$ for different $l / \lambda, n_{\mathrm{T}}=\frac{3}{2} n_{\mathrm{R}}$

\section{CONCLUSION}

In this work we analyzed the asymptotic capacity of the dense multiple antenna configurations. For the point-topoint MIMO channel we have shown that in the absence of CSIT, the capacity is bounded and related to the ratio between the size of the antenna array and the transmit signal wavelength. The capacity grows unbounded if perfect CSIT is available. In case of the dense MIMO broadcast channel the per-user capacity goes asymptotically to zero for ZF-beamforming as well as for MMSE-beamforming.

\section{REFERENCES}

[1] C. E. Shannon, "A Mathematical Theory of Communication", The Bell System Technical Journal, Vol. 27, pp. 379-423, 623-656, July, October, 1948.

[2] G. J. Forschini and M. J. Gans, "On Limits of Wireless Communications in a Fading Environment 
when Using Multiple Antennas", Wireless Personal Communications, Vol. 6, No. 3, pp. 311-335, 1998.

[3] I.E. Telatar, "Capacity of multi-antenna Gaussian channels," Tech. Rep., ATT Bell Labs, 1995.

[4] T.S. Pollock, T.D. Abhayapala, and R.A. Kennedy, "Antenna saturation effects on dense array MIMO capacity," in IEEE Intl. Conf. on Commun., ICC'03, Anchorage, Alaska, May. 11-15 2003, pp. 2301-2305.

[5] E. T. Jaynes, "Probability Theory: the Logic of Science", Cambridge University Press, 2003.

[6] M. Guillaud, M. Debbah, A. Moustakas, "Maximum Entropy MIMO Wireless Channel Models", Submitted to IEEE Trans. on Information Theory dec. 2006, arxiv.org/abs/cs.IT/0612101.

[7] D. V. Voiculescu, "Free Probability Theory", American Mathematical Society, 1997.

[8] P. Viswanath, D. Tse, "Sum capacity of the vector Gaussian broadcast channel and uplink-downlink duality", Vol. 49, No. 8, pp. 1912-1921, 2003.

[9] G. Caire and S. Shamai, "On the achievable throughput of a multiantenna Gaussian broadcast channel", IEEE Trans. on Information Theory, Vol. 49, No. 7, pp. 1691-1706, 2003.

[10] V. L. Girko, "Theory of random determinants. Transl. from the Russian", Mathematics and Its Applications (Soviet Series), 45, 1988.

[11] A.M. Tulino, A. Lozano, and S. Verdú, "Impact of correlation on the capacity of multi-antenna channels," IEEE Trans. on Information Theory), Vol. 51, No. 7, pp. 2491-2509, 2005.

[12] P. Dent, G. E. Bottomley and T. Croft, "Jakes fading model revisited", Electonics Letters, Vol. 29, No. 13, June 1993.

[13] J. W. Silverstein and Z.D Bai. "On the empirical distribution of eigenvalues of a class of large dimensional random matrices," Journal of Multivariate Analysis, 54: 175-192, 1995

[14] A. M. Tulino, S. Verdú, "Random Matrix Theory and Wireless Communications", Now Publishers, Vol. 1, Issue 1, 2004

[15] Z. D. Bai and J. W. Silverstein, "No eigenvalue outside the support of the limiting spectral distribution of large dimensional sample covariance matrices", Ann. Probab. Vol. 26, No. 1, pp. 316-345, 1998.

[16] L.Schumacher K.I. Pedersen and P. E. Mogensen, "From antenna spacings to theoretical capacities Guidelines for simulating MIMO systems", PIMRC 2002

[17] L. Zheng and D. Tse, "Communicating on the Grassmann Manifold: A Geo- metric Approach to Noncoherent Multi-antenna Channels", IEEE Transactions on Information Theory, Vol. 48, February 2002, pp. 359-383.

[18] M. Debbah and R. Müller, "MIMO Channel Modeling and the Principle of Maximum Entropy", IEEE Transactions on Information Theory, Vol. 51, May, No. 5, 2005

[19] E. Wigner, "Random Matrices in Physics", SIAM Review, Vol. 9, No. 1, January, 1967.

[20] R.M. Gray, "Toeplitz and Circulant Matrices: A Review", Foundations and Trends in Communications and Information Theory, 2006, Vol. 2, No. 3, pp. $155-239$

[21] D. Tse and P. Viswanath, "Fundamentals of Wireless Communication", Cambridge University Press, 2005.

[22] C. Peel, B. Hochwald and A. Swindlehurst, "A vector-perturbation technique for near-capacity multiantenna multiuser communication - Part I: Channel inversion and regularization", IEEE Trans. on Communications, Vol. 53, No. 1, pp. 195-202, Jan. 2005. 